\title{
A Relation-Based Product Model Suited for Integrating Design and Manufacturing
}

\author{
A.J. van der Net, W.A.H. de Vries, F.L.M. Delbressine, A.C.H. van der Wolf (1), \\ Eindhoven University of Technology, Eindhoven, Netherlands
}

Peceived on January 9, 1996

\begin{abstract}
In this paper a model of the design process is proposed. based on manufacturable design transformations. The product model enables manufacturability checking while designing. The model is based on relations between geometrical entities, with a tolerance model as an inseparable part. When performing for instance assembly analysis, creating numerical code for a machining centre. or command code for a Co-ordinate Measuring Machine. the product description is interpreted for implications in those specific applications. The authors demonstrate a method to reason with tolerances in several stages in design and manufacturing. A typical example is used to illustrate the method.
\end{abstract}

Keywords: $\quad$ product modelling, tolerancing, computer integrated manufacturing

\section{Introduction}

Current market demands comprise high quality, great product variety, and low product cost. To meet these demands, designers need suitable methods and tools. Checking manufacturability of a product in an early phase decreases the number of iterations and therefore reduces total throughput time. Two possible ways of implementing manufacturability checking exist: fearure recognition and feature based design.

In feature recognition, volumetric models are translated into "manufacturing features" to automate process planning of completed designs, as in for instance ICEM PART [Erve 88]. Fealure based design uses pre-defined geometrical shapes as functional elements to create a product description. If the features are related to manufacturing operations, on-line verification of manufacturability enables "right first time" manufacturing and automated process planning

Both the feaiure based design and feature recognition concept have resulted in tools trying to overcome the gap between the designing and manufacturing of discrete products that have a reiatively simple geometry and are manufactured using complex technologies

Most of today's CAD/CAM systems cannot keep track of limitations related to manufacturability. They rave emerged from the capabilities of computers to automate the drawing process Incorporating manufacturing knowledge in existing CAD systems, without fundamentally changing their concepts, will not render sufficient results.

Problems arise when modelling tolerance relations and assembly operations: tolerances should b-: more than just attributes, while assembly operations should surpass the "bill of material" stage. The design and manufacturing processes should be thoroughly studied to find fundamental concepts and models suitable for integrating design and manufacturing. Such a concept is proposed in this paper and described on the basis of a new product modei.

\section{Proposed product model}

A product model is used to satisfy three basic needs:

- Create a consistent product description for all stages in design and manufacturing;

- Capture and record the "design intent",

- Enable manufacturability analysis while designing.
A product model should be suited for describing information in all stages of the design and manufacturing process. This informaticn is preierably located in a single consistent product description Available information can be interpreted conform the neeas of the different stages Multiple models describing the same information calse problems of consistency

A design process rescilts into a description of a product accomplishing certain functions. This "design intent" is more easily captired when it is specified during the design process, instead of being hidden in for instance a dimension or tolerance value of the product. Functional relations, such as "join" or "rotate abour", shouid also be included in the product model. Although still incomplete ir capturing non-geometrical functions, a greater amount of information is available in the design process. A more complete product model also enables fast redesign and results into improved knowledge of the resulting manufacturing consequences. A design process model should enable capturing and recording the designers intent at different levels, making this information available during the entire process. This is critical since not all information needed can be deduced from a volumetric representation only [Erve 88).

To manufacture a product "right first time", the designers' decisions are verified at the moment they are made. Manulacturablity analysis includes selection of suitabie manufacturing processes given the geometry and tolerance values, or the ability to assernble a product given the relations between various parts. ine results of manufacturability checks are reported to a designer at the moment manufacturaisility problems can be predicted In conventionai design, a designer is free to create designs that cannot be nianufactured with a given set of manufacturing techriques. Manufacturability checking while designing requires the use of design operations that can be separately checked.

\subsection{Model basics}

The proposed model is used in an environment where prisnatic products are designed usirg volumetric descriptions. Products are manufactured on numerically controlled machines and quality assurance is performed using co-ordinate measuring machines. In numerous science fieids, such as materials engineering. computer science, and physics, the concept of states and state transitions is well known. $\dot{A}$ state transition uses a given state as 
input and it results into a new siate. The concept of states and state transitions can be applied to describe the design of mechanical products: "design states" and "design transformations" (Figure 1).

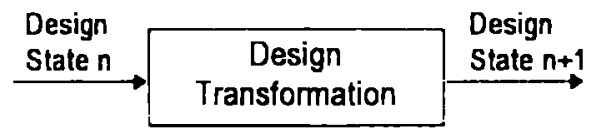

Figure 1: Design process

A design transformation is specified by a designer. When specifying a design :ransformation, information is collected and processed concerning geometry, functions and menufacturing iechniques. As stated before. in conventional design, a design transformation is not explicitly known or recorded. Generally, the final product, i.e. Its nominal geometry and tolerances, is specified using drawings, wire frame models, surface models, or volumetric models

If design transiormatiors are limited to manufacturable design transformaticns only, the resulting design state is manufacturabie. provided the design process is initialised using a manufacturable initial design state. Manufacturability can thus be ensured by introducing restrictions to the design transformations, originating from limitations of the avaliable manufacturing processes

The concept of manufacturable design transformations is translated into a set of operators and cperands. A designer uses these cperators and operards to specify a desired design transformation. The current design state is one of the operands needed. The other operand depends upon the type of operation. In case of an assembly operation, the other operand is a design state as well. When designing single parts, the second operand is a 'design cbject" (Figure 2). A design object can be made speciíc for a manufacturing technique, a function, or a modification of product geometry or material properties. A design object therefore has numercus different implementations

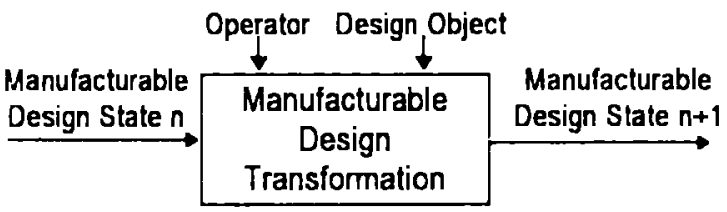

Figure 2: Operator and operands in design

Examples of design objects are Manufacturable Cbjects aric Primitive Objects (Figure 3). By using MO's or PO's in a design transformation, the geometry of the design state is modified.

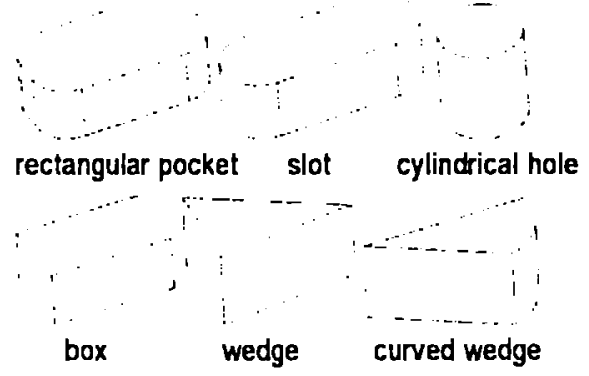

Figure 3: Examples of design objects

A Manulacturable Object is the "design and manufacturing pianning colinterpart of the application of a combinalion of one or more tools, machires, and sut-ups in the manufacturing phase; as introduced in [Deibressine 89]. Manufacturable Objects are mapped upon material removal manufacturing operations (e.g. drilling, milling)

The number of available design transformations can be increased by finding new ways of mapping design objects and operators onto manufacturing lechniques, thus shitting the limits of the designers' capabilities An example is demonstrated in Nries 95], where Primitive Objects are used with "add" and "subtracr" operatcrs while still guaranteeing manufacturability

Reference elements are an essential part of the model, of which design cbjects are composed. The volume of a design coject consists of the space enclosed by its reference elements. Relerence elements serve three purposes:
1. Captire relations that specify position, criertation anc dimersions of object geometry.

2. Capture tolerances

3. Capture assembly relations, such as "join" and "rotate about" Three types of reference elements can be identified a straight edge. a planar face, and a cylindrical face. A support pcint, a rormal- or direction vector, and a radius specify these eiements Not ail elemerts are used fer the above mentioned purposes Ar. edge is reeded for locating and dimensicring. Since an edge cannot be directly measured however, it is preferably not used icr icleraricing pliposes. For creating relations that include :olerances the faces from which the edge originates should be used insiead Figure 4 shows an "exploded view" of two design objects with their reference eiements (the straight edges are not depicted here)

Figure 4: Siot and cylindrical hole reference elements Reference elements are extended with tolerance information Sesices a nominal reference element (ype, radius. position, and orientation), two kinds of tolerance information are recresented

- The stated form tolerances and roughness value:

- Tolerance information resulting from reasoning with the stateo tolerances, as will be explained later

in sut:sequent design transformations, reference elements of design objects are specified using relations with reference elements of previously applied design objects. These elations include the stated iolerances (tolerances using two faces). Design object geometry and location are inlerred from the relationships. The design object geomety, location, and the knowledge incorporated in the design object specify the design transformation

For solid modelling operations this reference element based geometry can be used, including the results of tclerance interpreiation The desigr, state solid is composed using cimensions and locations that are not resulting from rominal values only.

A collection of subsequent design transiormations represents the design history. This collection is called a "design tree". A design tree has branches, since modelling assembly operations is possible by stating relations between reference elements of multiple parts

If geometry is to be inferred from relations a designer specifies, the nunber of relations must be sufficient. Furthermore. conflicting relations are not allowed. A method to determine if the given constraints are correct and complete has been developed. This method is based upon decomposition of a design object in three orthogonal directions that are local to the design object [Net 94]. For each of these directions the number and type of reference elements specified by the giren relations is determined. Only a iimited number of object type specific combinations is valid.

A design transformation is mapped upon manufacturing operations to create the physical counterpart called manufacturing state. While mapping a collection of design transformations onto manufacturing operations, nominal geometry, tolerances, avaiiable resources, and material properties are taken into account [Delbressine 90]. (Vries 95]. Based upon the available data, process planning is performed

\section{Tolerancing}

Using tolerances in an integrated design and marufacturing environment requires the tolerance specification to be interpreted to the specific rieeds of each design or manufacturing stage $A$ toierance model should subport multiple interpretations: for instance in process planning, assembly analysis, or quality assurance Current tolerance models cannot meet these requirements. partly because they are based on drafting lechniques. The transition from drawings io volumetric models and the use of modern measuring machines necessitates a more suitable tolerance reoresentation 
3.1. Tolerance model basics

Several tolerancing principles are currently available and standardised to assist in a description of the stated tolerances. Among the ISO 8015 standard Maximum Material Condition (MMC) and Least Material Condition (LMC) a combination of the two is described: the envelope requirement [Henzold 95]. The envelope requirement specifies the face to be contained in an envelope which is located in the vicinity of the nominal face (Figure 5). Since this description is closest to the actual surface deviation, the envelope requirement is chosen for the proposed tolerance model. The envelope requirement is applicable for both planar and cylindrical faces, the reference elements in the proposed product model.

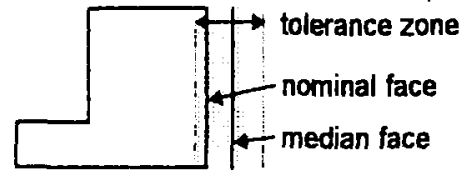

Figure 5: Proposed envelope requirement principle.

As stated before, tolerance information is part of a reference element. Using the envelope requirement principle, this information can be represented by creating a median face and a tolerance zone for each of the reference elements of a design object (Figure 6)

tolerance zone boundaries median face

Figure 6: Tolerance information

The proposed tolerance model is based on four principles:

- Tolerances specify restrictions a product should conform to;

- Tolerances are not hierarchical;

- The strictest tolerance for a single face is decisive;

- Tolerances are interpreted using the principle of dependency.

In these definitions, the proposed model deviates from the ISO 8015 model. In ISO the tolerances describe how a manufactured product can deviate from its nominal shape and still be valid [Henzoid 95]. A workpiece should conform to all tolerances at their extreme values, while hierarchy rules state the form tolerance to be the smallest and the size tolerance the largesi (Figure 7).

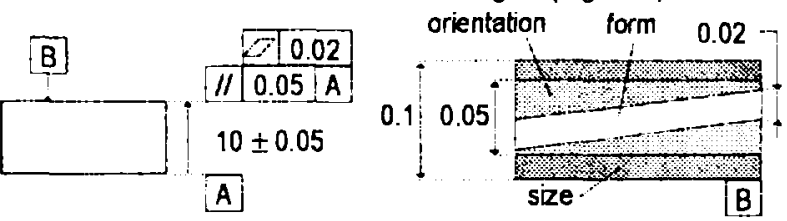

Figure 7: Tolerance hierarchy

A designer states tolerances resulting from functional requirements. From the designers' point of view, these requirements are not subject to any hierarchy. The proposed tolerance model therefore interprets tolerances as requirements which have to be met to ensure the functionality of a product. The strictest tolerance for a single face, which can be a size, form, orientation, or location tolerance, defines the surface deviation. This interpretation results into for instance a flatness tolerance sometimes exceeding the size tolerance, whereas in ISO this is not allowed.

The absence of hierarchy conforms with the designers' intent. Furthermore, it improves the ability to reason with the stated tolerances in both the design and manufacturing phase, whereas conventional tolerancing is mainly suitable for measuring purposes. In accordance with ISO, the model defines the envelope requirement principle as being tolerance dependent. When inspecting a single face not only size and form tolerances have to be checked, but also whether the envelope requirement is met. Figure 8 illustrates a product which satisfies the principle of independency, whereas the envelope requirement is not met.

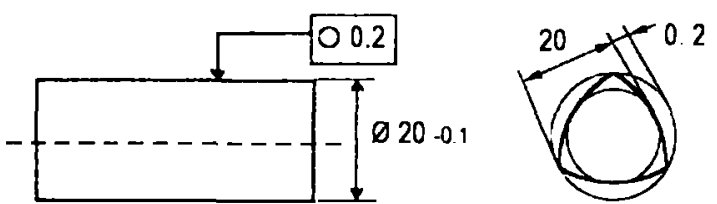

Figure 8: Principle of independency

When tolerancing independently, the stated tolerances are more easily met and inspected. For a designer however, it is more difficult to interpret their physical implications, so the principle of dependency is more suitable. This is especially important when using fits in assembly operations, since both size and form tolerances influence the fit. Therefore, taking assembly modelling into account, the principle of dependency for tolerances is preferred. 3.2. Reasoning with tolerances

Some assumptions are made when reasoning with tolerances:

- Tolerances are not directed; no datum plane is indicated;

- Reasoning is based on equal "manufacturing effor"t.

As stated before, reference elements are used to specify relations, which may be extended with tolerances. Relations between reference elements are not directed, i.e., no datum plane is indicaled. The absence of a datum plane enables reasoning with tolerances while taking manufacturing into account, without being detrimental to functionality. A tolerance relation affects both faces Reasoning with stated tolerances is based on equal manufacturing effort for both faces. To minimise total manufacturing effort, the face requiring the least effort is decisive if a relation concerns two faces. The other face is manufactured using equal effort.

To enable reasoning with tolerances based on manufacturing effort, manufacturing knowledge must be present in the design stage. The manufacturing effort for each tolerance relation is expressed in quality numbers [Vries 96]. Higher numbers indicate a mapping to less expensive manufacturing processes. When calculating quality numbers, the tolerance relation type and the dimensions of the faces involved are taken into account. Since for instance paralielism tolerances are easier to meet compared to size tolerances of equal tolerance value, they result into higher quality numbers

Distribution of a tolerance-zone is illustrated in Figure 9. A tolerance zone of $0.04 \mathrm{~mm}$ is distributed to create an equal quality number for both faces, while the total remains $0.04 \mathrm{~mm}$. Since the faces are not equal in dimension (relevant length), equal manufacturing effort introduces non equal distribution of the tolerance-zone 0.01 and $0.03 \mathrm{~mm}$

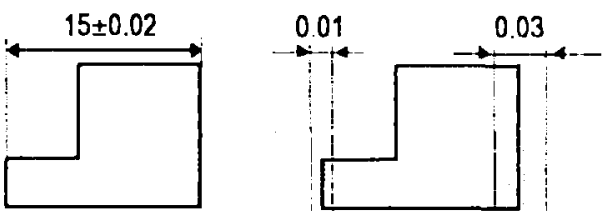

Figure 9: Distribution of a tolerance zone

If a face is used in multiple tolerance relations, the strictest requirement is decisive. If a face is modified, the tolerance-zone distribution has to be recalculated. The tolerance-zones of other relations are redistributed. in Figure 10, two tolerance relations are stated. These relations are both distributed according to equal manufacturing effort, resulting into two tolerance zones for the right plane Relation " $\mathrm{B}$ " is decisive for the right plane $(0.017 \mathrm{~mm})$. Since the right face is manufactured to satisfy the strictest tolerance relation, the left face can be manufactured with less effort the tolerance zone increases to $0.023 \mathrm{~mm}$ )

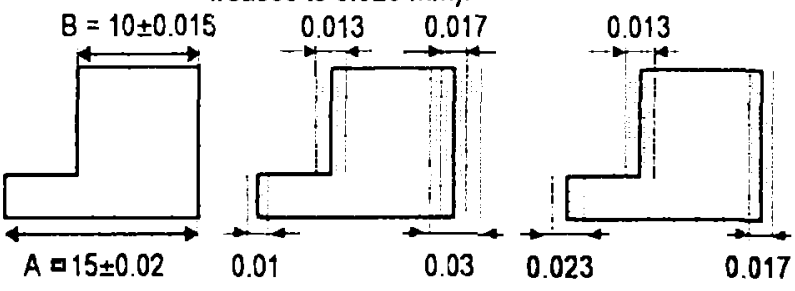

Figure 10: Redistribution using smallest tolerance zone 


\subsection{Interpretation of tolerances}

The siated tolerance can be interpreted serving difierent purposes In assembly, fil analysis is possible by checking for envelope inieraction When using surface treatments like etching and chemical vapour deposition, the gecmetry can be adapted by offsetting ail affected median faces and decreasing the tolerancezore widths with the suriace treatment deviation. In process pianning the stated tcierances are used to cincose suitable manufacturing lecriniques and for set-up analysis. When veritying geometry using a co-ordinate measuring machine, a surface is measured ard checked against the envelope

\section{Example}

Figure 11 depicts an examole cesign tree This cesign tree is a recorcing of design transformations to modify the geometry of three paris (cylincer, piston. piston-rod) and assembly operations

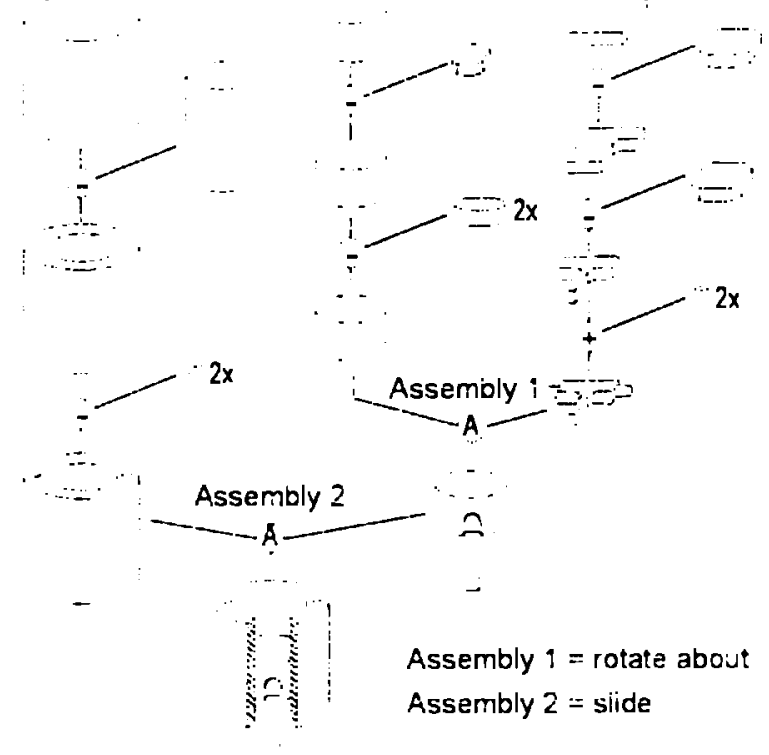

Figure 11: Design iree example

Part of this design tree concerns a slot in the piston. This design transformation is specifed using reations (Figure 12)

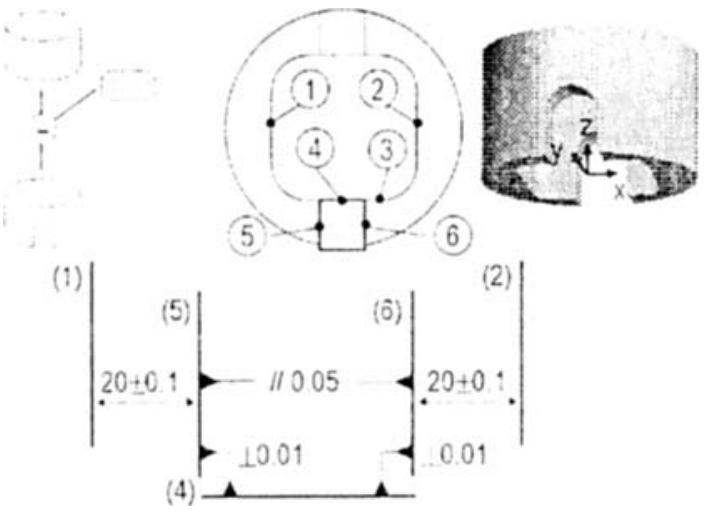

Figure 12: Applied slot in piston

Quality numbers are calculaled for each of the tolerance relations (Table 1). The lowest quality number, indicating the highest manufacturing effort, is ciecisive

\begin{tabular}{|l|l|l|l|l|l|}
\hline & face 1 & face 2 & face 4 & face 5 & face 6 \\
\hline $20+0.1$ & 9.6 & & & 96 & \\
2010.1 & & 9.6 & & & 96 \\
$1 / 0.05$ & & & & 8.7 & 8.7 \\
10.01 & & & 5.3 & 5.3 & \\
10.01 & & & 5.3 & & 53 \\
\hline
\end{tabular}

Table 1: Guality numbers

From these numbers it is concluded that the parallelity relaticn (quality $3 . \bar{T}$ ) is not useful, sirce it is already met; faces 5 and $\hat{b}$ are manufactured to meer the perrencicularity relation (quality 5.3).
Based upon the decisive quality rumbor, the tclerance zone distribution is calculated The tolerances of size $(20.01)$ describe a tolerance zone of $0.2 \mathrm{~mm}$ that can be distributed among faces $1-5$ and 2-6 respeclively. Since perpendicularity requires quality number 5.3, which corresponds with a colerance zone of $0.01 \mathrm{~mm}$, the remaining part of the size tolerances form the iclerance zones of faces 5 and 5 respectively (Figure $i 3$;

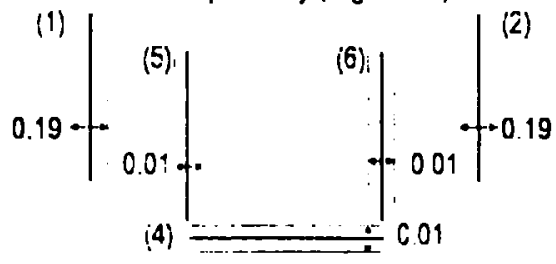

Figure 13: Tolerance zones

After distribution of :olerances. quality numbers are recalculated Faces 1 and 2 now are of quality 11 ( 9.0 before) These faces can be manufactured by rough milling only instead of rough and finish milling, making it less expensive. The designers intent is still me: since the other faces, 5 and 6 , are manufactured by grirding to meet the perpendicularity relation (quality 5.3).

The quality numbers can be used to show the impact of a stated tolerance value. if the perpendicularity is modified 100.02 (quality 7 ), faces 4 5, and 6 need no grinding operation, which lowers throughput ime ard product costs Because of this type of tolerance reasoning it is pcssibie to automarically suggest such modifications to the designer.

\section{Conclusions}

it can be conciuded that the proposed product model is suitable for an ntegrated environment enablirg manufacturability aralysis while designirig. The procosed product model is able to incorporate no! only the geometry and the tolerance relations, but also higher-leve information This better enables the capturing "design intent" ano opens the possibility to include assembly relations in one consistent product description

The tolerance model included in this product model founds on the prirciple of cependence and an equal 'marufacturing effort' and can be used in for ooth measurement. mantifacturing. and design aralysis By using empiricai knowledge of manufacturing processes in the design stage, the principle of equal "manufacturing effort" enables more economical production

\section{References}

[Boerma 90] Boerma, J.R. 1990. The design of fixiures for prismalic parts Ph.D. thesis, University of Twente

[CIRP 93] 1993, Proceedings of 3" CIRP Seminars on Computer Aided Toleraricing, Eyroiles, Paris

[Delbressine 89] Delbressine. F L.M. 1989, On the integration of design and manufacturing, $\mathrm{Ph} \mathrm{D}$. thesis, Eindhoven University of Technology, Eindhoven.

[Erve 88] Erve, A.H. van 1, 1988. Generative compuler aided process planning for pant manufacluring: An expert system approach, Ph.D. thesis, University of Twente, Enschede

[Fazio 93] Fażio, T.L de, et al 1993 A protolype of feature-based design for assembiy. J. of mech design, Vol. 115, pp. 723-734

[Henzold 95] Henzold, G, 1995, Handbook of Geometrical Tolerancing. Design Manufacturing and Inspection, Joln Wiley \& Sons. Chichester

[Net 94] Net. A.J. van der, 1994, in;;ilicit locating for specifying geometrical obiects in IDAl, Eindhoven University of Technology. Stan Ackermans Institute, Einchoven

[Turner 93] Turner, JU., 1993, A keasibility space approach ior automated' tolerancing. J. of Eng. for Iricisiry, Vol. :15. 341-346

Nries 95] Vries, 'N.A.H. de, Delbressine FL.M. Wolf, A.C.H. van der, 1095. Guaranteeing Manufacturability of CSG operations Annais of the CIRP Vol 44/1, pp $\$ 7-100$

Nries 96] Vries. WAH de, 1996. Manufaclurable Design Ooerations, Ph J. Thesis. Sindhoven University of Technoicgy, Eindhoven (to be cutlisined) 